\title{
Oncolytic Nanoreactors Producing Hydrogen Peroxide for Oxidative Cancer Therapy
}

\author{
Seong-Min Jo, Frederik R. Wurm, ${ }^{*}$ (i) and Katharina Landfester*(;) \\ Max Planck Institute for Polymer Research, Ackermannweg 10, 55128 Mainz, Germany \\ Supporting Information
}

ABSTRACT: In situ generation of anticancer agents at the place of the disease is a new paradigm for cancer therapy. The production of highly potent drugs by nanoreactors through a facile synthesis pathway is demanded. We report an oncolytic nanoreactor platform loaded with the enzyme glucose oxidase (GOX) to produce hydrogen peroxide. For the first time, we realized a core-shell structure with encapsulated GOX under mild synthetic conditions, which ensured high remaining activity of GOX inside of the nanoreactor. Moreover, the

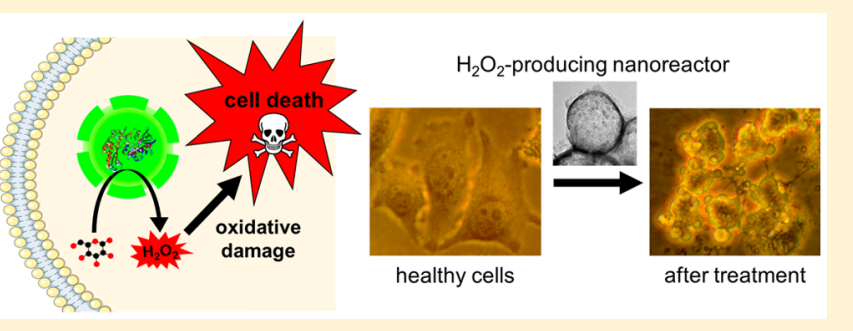
nanoreactor protected the loaded GOX from proteolysis and contributed to increased thermal stability of the enzyme. The nanoreactors were effectively taken up into different cancer cells, in which they produced hydrogen peroxide by consuming intracellular glucose and oxygen, thereby leading to effective death of the cancer cells. In summary, our robust nanoreactors are a promising platform for effective anticancer therapy and sustained enzyme utilization.

KEYWORDS: Nanoreactor, oxidative cancer therapy, enzyme reactions, glucose oxidase, enzymatic therapeutics, enzyme protection

Ta n contrast to direct treatment of drugs, the concept of a "drug factory" had been proposed as a next generation of medication. It is a chemical conversion of prodrugs to active drugs or synthesis of the drugs via nanoreactors in the body, which is inspired from plasma cells. ${ }^{1-4}$ However, the prodrug conversion strategy is plagued by often expensive and unfamiliar prodrugs. ${ }^{5}$ The synthesis of entire anticancer drugs through a nanoreactor as a drug factory is another attractive strategy but includes complicated synthetic pathways, the requirement of continuously supplying the precursors, and unexpected side-effects by side-products, which renders this idea highly challenging. ${ }^{6,7}$ Thus, production of easier drugs by the nanoreactors, which relies on simple synthetic procedures and facile to supply precursors, is of great interest. Moreover, the production of the drugs at the place of the disease (e.g. inside the tumor) is believed to be a "smart disease care" for effective medication. Here, we present enzyme-loaded nanoreactors that not only protect the enzymes but also produce hydrogen peroxide by consuming glucose and oxygen inside of cancer cells. We achieved a one-step and glucose-derived production of hydrogen peroxide inside of cells and an effective killing of cancer cells in this study.

Hydrogen peroxide has been paid attention to cancer therapeutics due to its reactivity and since it has critical influences on cellular metabolism. ${ }^{8}$ Hydrogen peroxide results in serious damage such as generating oxidized nucleic acids (e.g., 8-oxo-2'-deoxyguanosine and 5-methylcytosine), protein deactivation, and lipid peroxidation. Persistent generation of hydrogen peroxide can deplete antioxidant scavengers (e.g., glutathione) in cells, thereby causing necrosis due to oxidative stress. ${ }^{9,10}$ Moreover, an aberrant amount of hydrogen peroxide gives rise to unexpected activation of proapoptotic proteins such as B cell lymphoma 2 associated X (BAX) and caspase3. ${ }^{11,12}$ Consequently, this fatal damage and intracellular disturbances end up with necrotic/apoptotic cell death. ${ }^{13}$ As "oncolytic hydrogen peroxide" is easily producible through a one-step reaction of glucose oxidase (GOX) by consuming glucose and oxygen, it is one of the ideal candidates for an anticancer agent that can be produced by a "drug factory" in the body.

As most enzymes including GOX are immunogenic, expensive, instable in an in vivo environment, and cannot enter into cells, nanoencapsulation is highly desired. ${ }^{14}$ Moreover, enzyme reactions in confinement are more efficient compared to solution conditions. ${ }^{15-18}$ Several nanoreactors with enzymatic production of hydrogen peroxide and their cancer treatment have been proposed in recent years. ${ }^{19-27}$ GOX was loaded to polymeric or lipid nanoreactors, expecting oxidative stress, glucose starvation, or hypoxic effects to kill cancer cells. ${ }^{19-23}$ Further pioneering works presented the synergetic cytotoxicity of glucose starvation and nitric oxide or the photodynamic effects by immobilized GOX on the surface of silica nanoparticles. ${ }^{24,25}$ Nevertheless, those two studies did not describe the encapsulation of GOX inside the nanoreactors. Moreover, most nanoreactors loaded with GOX suffered from low loading efficiency or decreased activity ${ }^{19-23}$ and were chemically and physically fragile. Protection of loaded enzymes against external harsh conditions (e.g., high

Received: October 15, 2019

Revised: November 27, 2019

Published: December 2, 2019 


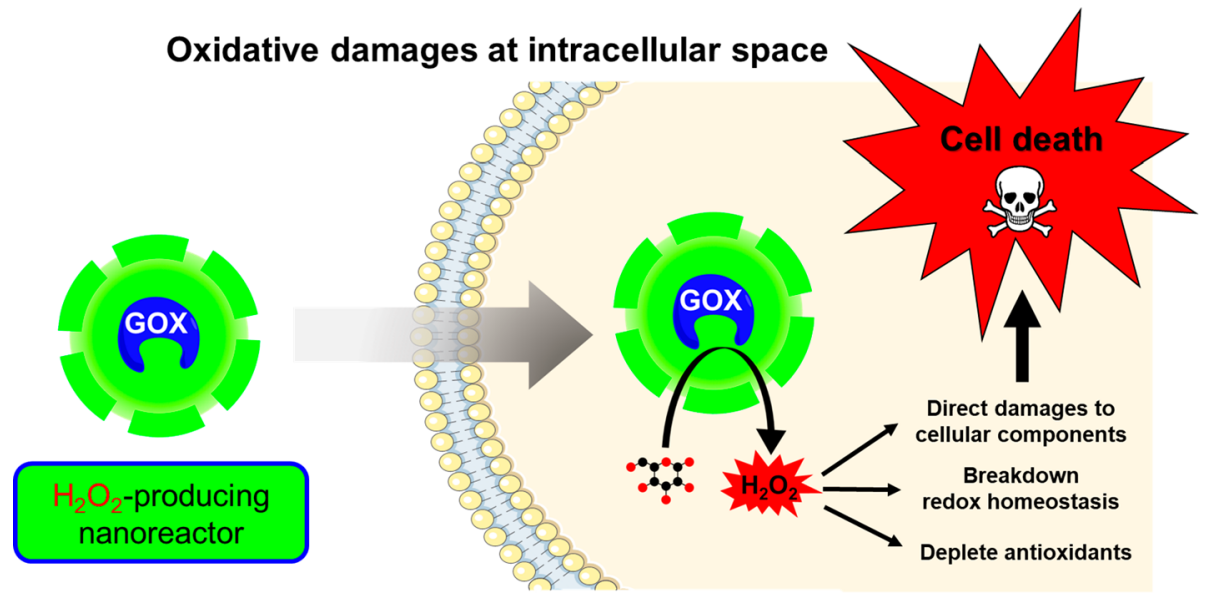

Figure 1. Concept of the glucose oxidase-loaded nanoreactors (GOX@NR) for cell death. Cellular uptake of the GOX@NR is enabled through phagocytosis, which intracellularly produces hydrogen peroxide by consuming glucose. Oxidative damage of cellular components, activation of apoptotic pathways, and depletion of scavengers, thereby leading to effective cell death.
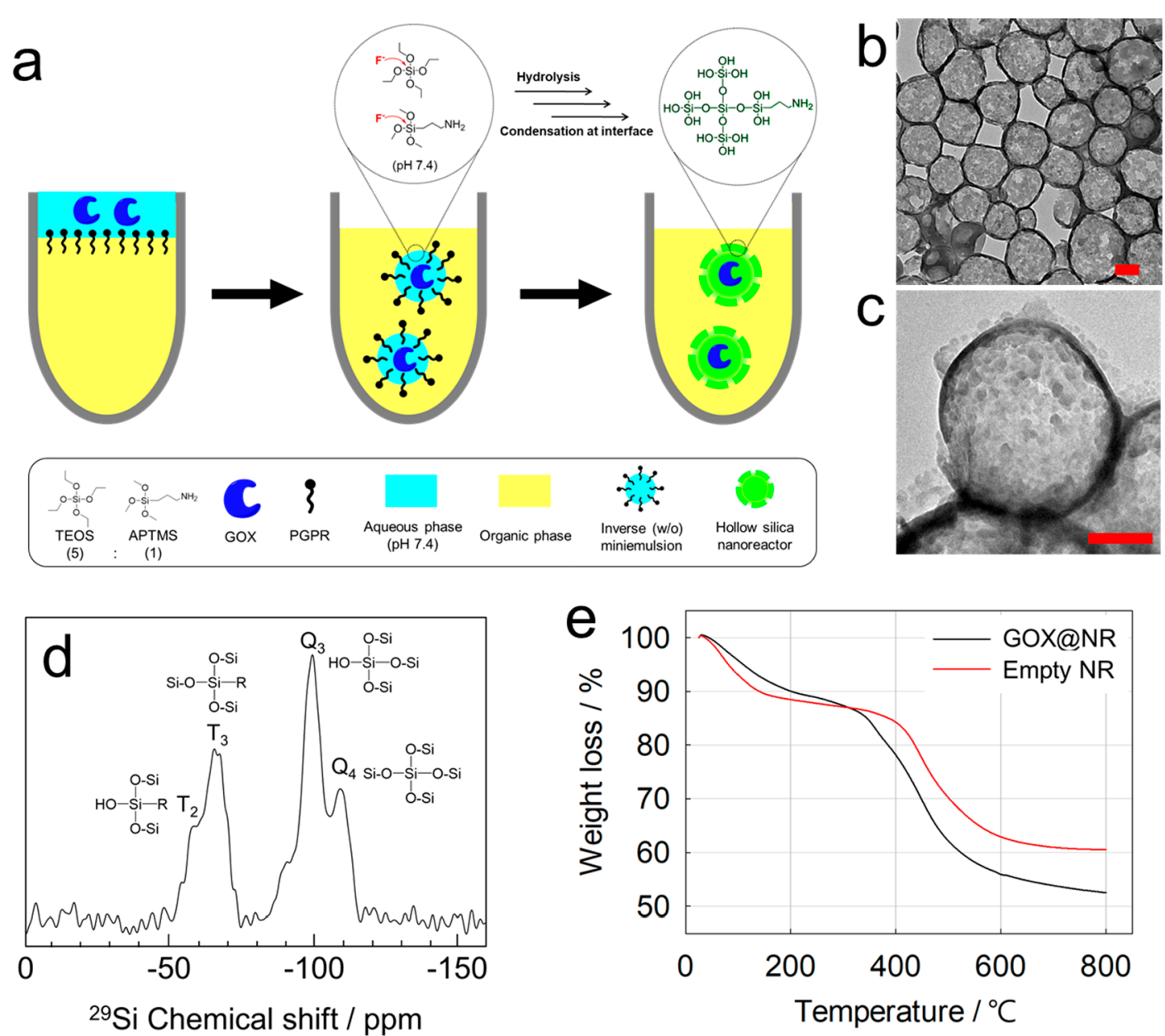

Figure 2. Synthesis and characterization of the hollow nanoreactor loaded with GOX. (a) Synthesis scheme: sol-gel chemistry takes place at the interface of an inverse (water-in-oil) miniemulsion by interfacial polycondensation of APTMS and TEOS precursors. (b, c) Transmission electron microscopic images of the silica nanoreactor with loaded GOX, $\times 10000$ (left) and $\times 40000$ (right) magnification, respectively. Red scale bars represent $200 \mathrm{~nm}$. (d) ${ }^{29} \mathrm{Si}$ solid state NMR spectroscopy of GOX nanoreactors composed of APTMS and TEOS. (e) Organic contents investigation of GOX@NR and plain nanoreactors by TGA.

temperature, proteolysis) was not explored for earlier reported nanoreactors. ${ }^{24,25}$ Therefore, it is necessary to develop a robust nanoreactor system with loaded enzymes for high performance, sustained availability, and stable immunoisolation.
Herein, we have designed oncolytic nanoreactors (NRs) that enzymatically produce hydrogen peroxide by consuming intracellular glucose and oxygen for oxidative cancer therapy (Figure 1). The GOX-loaded nanoreactors (GOX@NRs) were 

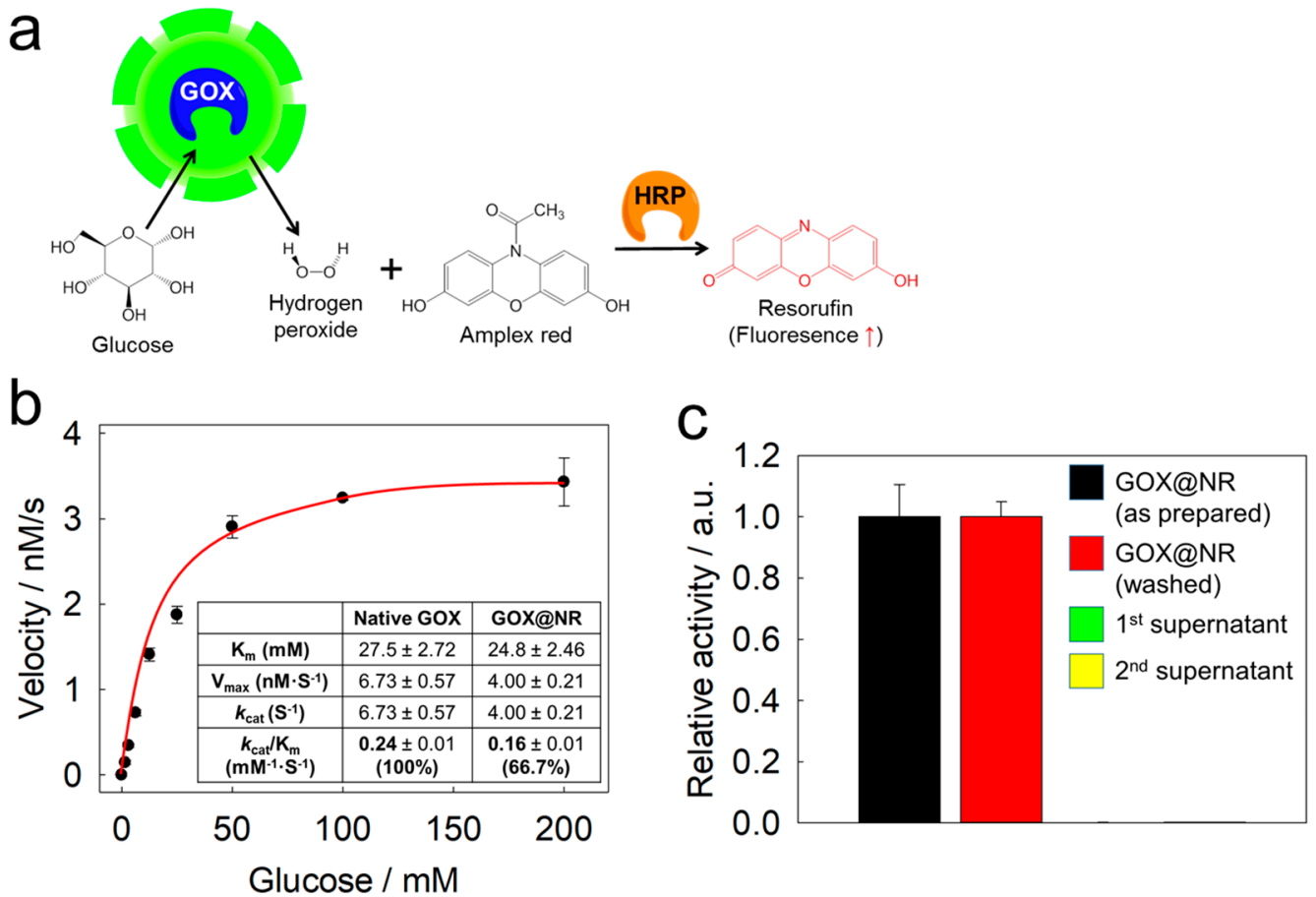

Figure 3. Enzymatic activity of GOX inside the nanoreactor. (a) Chemistry of the GOX assay. GOX produces hydrogen peroxide by consuming glucose. HRP converts Amplex red to resorufin in the presence of hydrogen peroxide, generating fluorescence at excitation $555 \mathrm{~nm}$ and emission $595 \mathrm{~nm}$. (b) Michaelis-Menten kinetics of GOX inside of the nanoreactor vs native GOX. (c) Leakage test of GOX from the nanoreactor was detected after washing. Negligible leakage (yellowish green and yellow bars) was observed.

prepared by a fluoride-catalyzed sol-gel process of silica. The hollow nanoreactors comprise an aqueous core and a permeable silica shell for small molecules like glucose or hydrogen peroxide. This is the first report to synthesize hollow and enzyme-loaded silica nanoreactors through fluoridecatalyzed and $\mathrm{pH}$ neutral conditions, ensuring high enzymatic activity after encapsulation. The silica shell of the GOX@NR effectively protects GOX against denaturation and increases the enzyme's stability. Glucose not only is an abundant nutrient but also exhibits higher uptake in most tumor cells due to their vigorous metabolism. We achieved an efficient anticancer therapy by the nanoreactors generating oncogenic hydrogen peroxide under physiological conditions without any additional prodrugs or xenobiotic precursors. We demonstrate that solely oxidative damage was enough for effectively killing cancer cells by intracellular production of hydrogen peroxide.

Results and Discussion. Design of Silica-Based Nanoreactors (NRs) with Loaded Glucose Oxidase (GOX). The aqueous-core/silica-shell nanoreactors with loaded GOX (GOX@NRs) were prepared via an interfacial fluoridecatalyzed sol-gel polycondensation in an inverse (water-inoil) miniemulsion (Figure 2a). For the miniemulsion, we used toluene as a continuous phase and a buffered aqueous phase (pH 7.4) containing GOX, aminopropyl trimethoxysilane (APTMS), and potassium fluoride. The biocompatible polyglycerol polyricinoleate (PGPR) was used as an emulsifier. After the formation of the miniemulsion, tetraethyl orthosilicate (TEOS) was added to the continuous phase to start the shell formation. Polycondensation of both silica precursors at the interface of the emulsion led to the formation of the nanoreactors with a hollow aqueous core and a permeable silica shell. We found that the APTMS plays a key role as a complementary catalyst in facilitating the interfacial polycondensation to form the cross-linked shell.
For hydrolysis and condensation of the silica precursors, we used an aqueous potassium fluoride $\left(\mathrm{F}^{-}\right)$solution acting as a catalyst for the sol-gel reaction. The fluoride anion can initiate a nucleophilic attack on $\mathrm{Si}$ of the silica monomer by an $\mathrm{S}_{\mathrm{N}} 2$ mechanism that catalyzes hydrolysis and polycondensation of the silica precursors to form silicon oxide (Figure 2a). Unlike conventional sol-gel chemistry that uses acidic or basic catalysts, the use of fluoride effectively catalyzes the sol-gel reaction at neutral $\mathrm{pH}(7.4)$, which preserves the enzymatic activity during the synthesis. We further proved that the fluoride catalysis is harmless for the GOX stability (shown below).

We observed the core/shell structure of the GOX@NRs by transmission electron microscopy (TEM) (Figure 2b). The thickness of the shell was estimated to be $19 \pm 3 \mathrm{~nm}$ by image analysis [calculated from a selection of 75 nanoreactors (Image s software, NIH)]. The size distribution of the GOX@NR was measured by dynamic light scattering ( $Z$ average: $378 \mathrm{~nm}$ in diameter) (Figure $S 1$ ). The $\zeta$ potential of the GOX@NRs was determined to be close to zero $(0.43 \pm$ $0.26 \mathrm{mV}$ ). In comparison to empty nanoreactors (without enzymes), no significant differences were observed ( $Z$-average $340 \mathrm{~nm}$, and $0.51 \pm 0.41 \mathrm{mV}$, respectively). Solid-state ${ }^{29} \mathrm{Si}$ NMR spectroscopy revealed a composition of silica precursors in the GOX@NRs, proving a successful incorporation of both precursors: $Q_{4}(19 \%), Q_{3}(37 \%), Q_{2}(7 \%)$, and $Q_{1}(0 \%)$ from TEOS and $T_{3}(12 \%), T_{2}(25 \%)$, and $T_{1}(0 \%)$ from APTMS, respectively, were quantified from the spectra (Figure $2 \mathrm{~d}$ ). The organic content of the GOX@NRs was determined by thermogravimetric analysis. Compared to empty nanoreactors, the GOX@NRs showed a further 8\% weight loss, which indicated the GOX content in the nanoreactors (Figure 2e).

Advantages of Fluoride Catalysis for the Preparation of GOX-Loaded Nanoreactors. We prepared two different 
nanoreactors, via the fluoride-catalyzed GOX@NR ( $\mathrm{pH} 7.4)$ and the ammonia-catalyzed GOX@NR ( $\mathrm{pH} 10.5)$, respectively. We found that the fluoride-catalyzed GOX@NR preserved a much higher enzymatic activity (66\%) than the ammonia-catalyzed GOX@NR (8\%). To explore the effects of each catalyst on GOX, we further performed an activity assay, circular dichroism (CD) studies, and thermal denaturation studies. The exposure to $0.1 \mathrm{mM}$ (as used condition in this study) and $10 \mathrm{mM}$ fluoride did not lead to any decreased activity for native GOX, whereas the GOX exposed to $3 \mathrm{M}$ (5\%) ammonia led to a significant loss of the catalytic activity (Figure S4). Regarding these different activities, the CD spectra (Figure S5) revealed that fluoride (both 0.1 and 10 $\mathrm{mM}$ ) did not change the secondary structure in the native GOX. In contrast, denaturation occurs with the GOX by exposure to ammonia, mostly decreasing the $\beta$-sheet structure from $29 \%$ (native) to $8.5 \%$ (denatured). Thermal denaturation showed that the exposure to fluoride (with 0.1 and $10 \mathrm{mM}$ ) has no negative effect on the thermal stability of the GOX, which was proven by the same melting temperature (55.5$55.9{ }^{\circ} \mathrm{C}$ ) (Table S1). Probably because of denaturation, a lower thermal stability was obtained from ammonia-exposed $\operatorname{GOX}\left(47.1^{\circ} \mathrm{C}\right)$.

Enzymatic Reactions inside of GOX@NRs. The enzymatic activity of GOX@NR was measured by an Amplex red fluorescence assay and compared to free GOX in solution (Figure 3a). Based on detailed Michaelis-Menten kinetics $\left(K_{\mathrm{m}}\right.$ $=$ Michaelis Menten constant), we found that $66 \%$ of the native enzymatic activity $\left(k_{\text {cat }} / K_{\mathrm{m}}\right)$ were retained after loading GOX into the nanoreactors (Figure $3 b$ ). This reduced enzymatic activity might be explained by a decreased accessibility of GOX inside of the nanoreactor or by partial denaturation during the miniemulsion process. Substrate affinity of the GOX $\left(K_{\mathrm{m}}\right)$ was not significantly different between native GOX and GOX in nanoreactors. In particular, the turnover number $\left(k_{\text {cat }}\right)$ on the reaction of the GOX in the nanoreactors was significantly reduced in comparison to the reaction of native GOX. We assume that the temporary product accumulation in the microconfined space (nanoreactors) occurred by delayed diffusion of the products, resulting in a stronger feedback inhibition that might lead to a reduced turnover number.

When the GOX@NRs were washed with additional water, no leakage from the nanoreactors was detected as no enzymatic activity was measured in the supernatant (Figure $3 \mathrm{c})$. This indicates that GOX $(150000 \mathrm{Da} ; 6.0 \times 5.2 \times 7.7$ $\mathrm{nm}^{3}$ ) cannot pass through the silica shell, while the substrate glucose (180 Da; $8.5 \AA$ in diameter) can easily penetrate the shell. These results are consistent with the average pore size of ca. $6.3 \mathrm{~nm}$ average pore size determined from BrunauerEmmett-Teller (BET) gas adsorption.

In addition, we found a diffusion effect of substrate and products to pass through a silica shell of the GOX nanoreactors. Different reaction kinetics were determined at low and high concentration of substrates. Unlike high (100 $\mathrm{mM}$ ) glucose concentration, the delayed diffusion effect was observed on the GOX reaction of the nanoreactors at low (1 $\mathrm{mM}$ ) glucose concentration (Figure S2). We could not observe this phenomenon from the reaction of both native GOX and surface-immobilized GOX. ${ }^{28}$ This result implies that changed kinetics of the GOX reaction in nanoreactors occurred due to diffusion effects.
Protection and Thermal Stability of Enzymes. Proteolytic resistance of loaded GOX in the nanoreactors was investigated by Proteinase K (EC 3.4.21.64; $28.9 \mathrm{kDa}$ ), which could not penetrate into the nanoreactors. Unlike bare GOX in solution, the GOX@NR did not lose the enzymatic activity in the presence of Proteinase K (Figure 4a). As different proteolytic
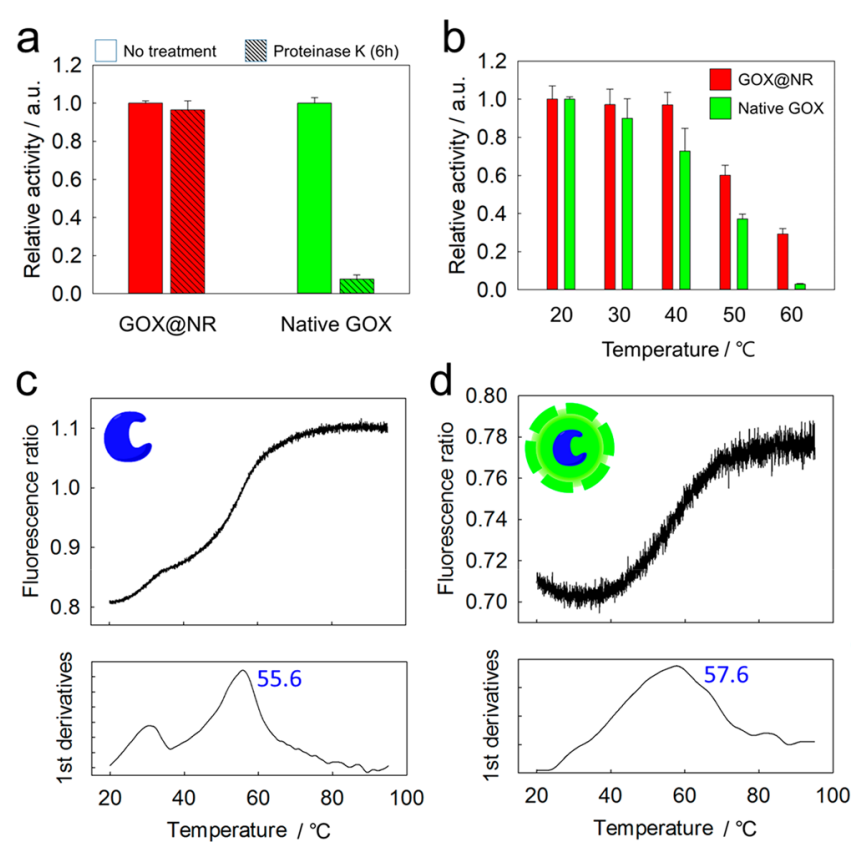

Figure 4. Protection of GOX inside of the nanoreactors. (a) Protease resistance of GOX in the nanoreactor and native GOX. (b) Thermal resistance of GOX inside of the nanoreactor and native GOX after exposure to temperatures from $20-60{ }^{\circ} \mathrm{C}$ for $10 \mathrm{~min}$. Thermal denaturation profiles of native GOX (c) and GOX inside of the nanoreactors (d), measured by NanoDSF.

enzymes are present in the in vivo environment, protection of GOX is an important prerequisite if considered as an anticancer drug. To explore applicability to the cellular environment, GOX stability from cell lysates was tested. GOX@NR kept their full initial activity, whereas the native GOX was remarkably reduced ( $76 \%$ of the initial activity) after exposure to the cell lysate (Figure S3).

Thermal stability of GOX was investigated by nanodifferential scanning fluorimetry (NanoDSF). This technique is based on changes in intrinsic fluorescence of aromatic amino acids in protein structures by thermal denaturation. The folded and unfolded proteins show a different emission at 330 and $350 \mathrm{~nm}$, enabling estimation of the melting temperature $\left(T_{\mathrm{m}}\right)$. Thermal denaturation profiles discovered that the GOX in the nanoreactors $\left(T_{\mathrm{m}}: 57.6^{\circ} \mathrm{C}\right)$ seems to be more stable than the native GOX $\left(T_{\mathrm{m}}: 55.6{ }^{\circ} \mathrm{C}\right.$ ) (Figure $\left.4 \mathrm{c}, \mathrm{d}\right)$. To confirm this increased stability, we also evaluated the change of the enzymatic activity after exposure from ambient temperature $\left(20^{\circ} \mathrm{C}\right.$ ) to $60{ }^{\circ} \mathrm{C}$ (Figure $\left.4 \mathrm{~b}\right)$. The native GOX quickly lost its activity after exposure to increased temperature. In contrast, GOX inside the nanoreactors proved to have a much higher thermal stability with residual activity of ca. $35 \%$ after exposure to $60{ }^{\circ} \mathrm{C}$ for $15 \mathrm{~min}$. In particular, GOX loaded in nanoreactors is more stable in the range $40-50{ }^{\circ} \mathrm{C}$ as well as native GOX, implying great advantages when combining with hyperthermia therapy. 

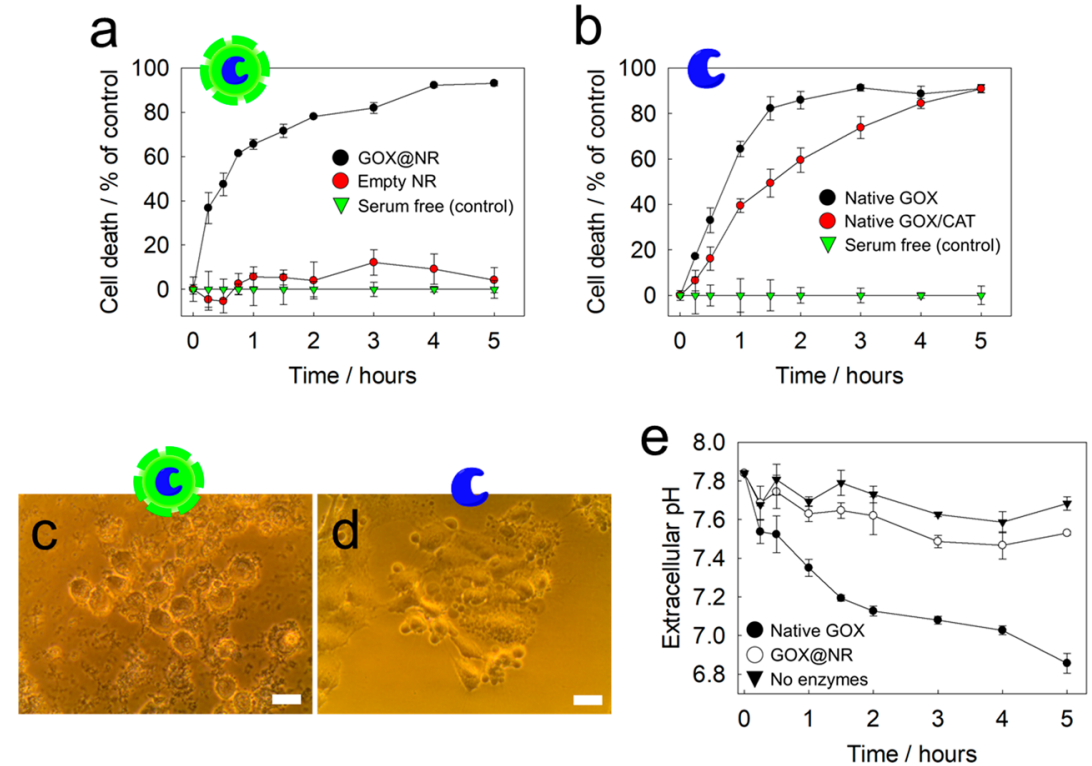

Figure 5. Nanoreactors with loaded GOX and native GOX-mediated cell death. Cytotoxicity assay of GOX-loaded nanoreactors (a) and native GOX (b) mediated cell death in time relapse $(n=3$, error bars $=$ SD). Microscopic image of dead cells by the GOX-loaded nanoreactors $(c)$ and native GOX (d) for a $3 \mathrm{~h}$ reaction. Scale bars represent $30 \mu \mathrm{m}$. (e) Monitoring changes in $\mathrm{pH}$ value of medium of outside cells during GOX reaction. Glucose concentration of $44 \mathrm{mM}$ was used for the time relapse assay $(n=3$, error bars $=\mathrm{SD})$. The HeLa cell was used for all assays.

Cytotoxicity Induced by Enzymatic Hydrogen Peroxide Production. We demonstrated the cytotoxic effect of hydrogen peroxide produced by GOX with a series of in vitro studies with HeLa cells (human cervical carcinomas) as model cancer cells. As a proof-of-concept, we treated native GOX or GOX@ NR with glucose $(44 \mathrm{mM})$ and incubated them with the cells. The cell viability was then monitored by a formazan/ dehydrogenase-based assay (CCK-8). In both cases, the enzymatic reaction produced $\mathrm{H}_{2} \mathrm{O}_{2}$ in the extracellular medium, and both native GOX and GOX@NR proved to have an effective cytotoxicity (Figure $5 \mathrm{a}, \mathrm{b}$ ). When catalase as a $\mathrm{H}_{2} \mathrm{O}_{2}$ scavenger was added additionally, a decreased cytotoxicity was observed (Figure $5 \mathrm{~b}$, red curve), indicating that the produced hydrogen peroxide is the major factor for cell death. In addition, we let the cells "starve" for a period of 5 $\mathrm{h}$ to deplete the intracellular glucose content, but no effects on cell viability were observed during this period of time (Figure S6).

Importantly, different effects of native GOX and GOX@NR on the cell death scenario were identified from microscopy images. In the case of native GOX-triggered cell death, we observed the generation of necrotic blebs on the cells, but overall they kept their original morphology (Figure 5d) as also reported earlier. ${ }^{29}$ During the native GOX reaction, the $\mathrm{pH}$ value of the medium (outside of cells) decreased due to the extracellular production of gluconic acid (Figure 5e), which supports the assumption of an extracellular enzyme reaction. In contrast, when GOX@NR was incubated together with the cells, almost no necrotic blebs but a certain shrinking of the cells was detected in the optical images (Figure 5c). Moreover, the extracellular $\mathrm{pH}$ value remained unchanged to the control (Figure 5e). This led us to the assumption that uptake of GOX@NR by phagocytosis leads to GOX reactions in the cytoplasm. When we treated GOX@NR or native GOX without addition of glucose, no change of the $\mathrm{pH}$ value was observed (Figure S7).
Even though a low level $\left(10^{-9}-10^{-7} \mathrm{M}\right)$ of hydrogen peroxide is naturally produced and essential for living cells, aberrant production can cause serious cytotoxicity to occur. ${ }^{30}$ Cells are afforded up to $10^{-7} \mathrm{M}$ of intracellular hydrogen peroxide by their own antioxidant defense system such as catalase and glutathione peroxidase. Below $10^{-6} \mathrm{M}$ concentration, cell cycle arrest can arise. A higher concentration $\left(10^{-4}\right.$ M) can lead to necrotic or apoptotic cell death. ${ }^{31,32}$ The mechanisms of the hydrogen-peroxide-mediated cytotoxicity were attempted to be understood by a few studies. The exposure of high concentrations of hydrogen peroxide (0.5$2.0 \mathrm{mM}$ ) can deplete the scavengers (i.e., glutathione) in cells, thereby causing necrosis due to oxidative stress., ${ }^{9} 10$ The Fenton-like reactions $\left(\mathrm{Fe}^{2+}+\mathrm{H}_{2} \mathrm{O}_{2} \rightarrow \mathrm{Fe}^{3+}+\bullet \mathrm{OH}+\mathrm{OH}^{-}\right)$ spawn another much more reactive oxygen species (ROS), the hydroxyl radical $(\bullet \mathrm{OH})$. It is highly reactive with major cellular components such as nucleic acid, proteins, and lipids, generating serious mutations or oxidative damage on cells. Furthermore, hydrogen peroxide triggers decreases in the production of superoxide $\left(\mathrm{O}_{2}{ }^{--}\right)$by inhibiting $\mathrm{NADH} /$ $\mathrm{NADPH}$-dependent oxidases. It occurs that augmentation of the reduction state of the intracellular milieu is followed by eliciting mitochondrial translocation of BAX (B cell lymphoma 2 (BCL2) associated X), and eventually leading to apoptosis. ${ }^{11}$ Hydrogen peroxide also plays an important role in the activation of the caspase- 3 signaling pathway, which leads to apoptosis. ${ }^{12}$ Although some cancer cells upregulate the resistance against exogenous hydrogen peroxide (e.g., membrane-bound catalase), an excessive and aberrant amount of hydrogen peroxide inevitably leads to cell death. ${ }^{33}$

In our previous experiments, both native GOX and GOX@ NR led to a high degree of cell death by either extracellular or intracellular production of hydrogen peroxide. To evaluate the potential as an intracellular drug factory, we first tried to deliver the native GOX (or GOX@NR) under glucose-free conditions, later adding glucose to the cells, and then evaluated the oncolytic efficiency (Figure 6a). In this experiment, any 
a

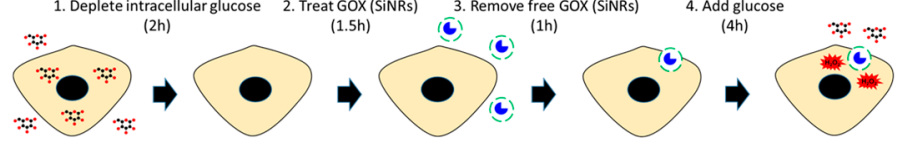

b
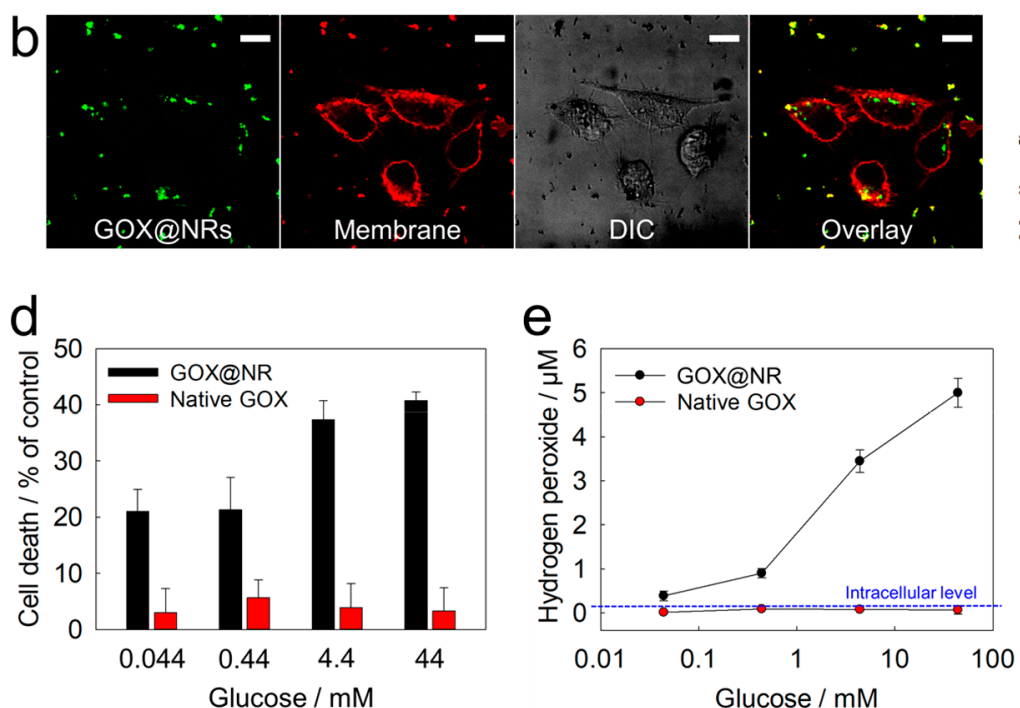
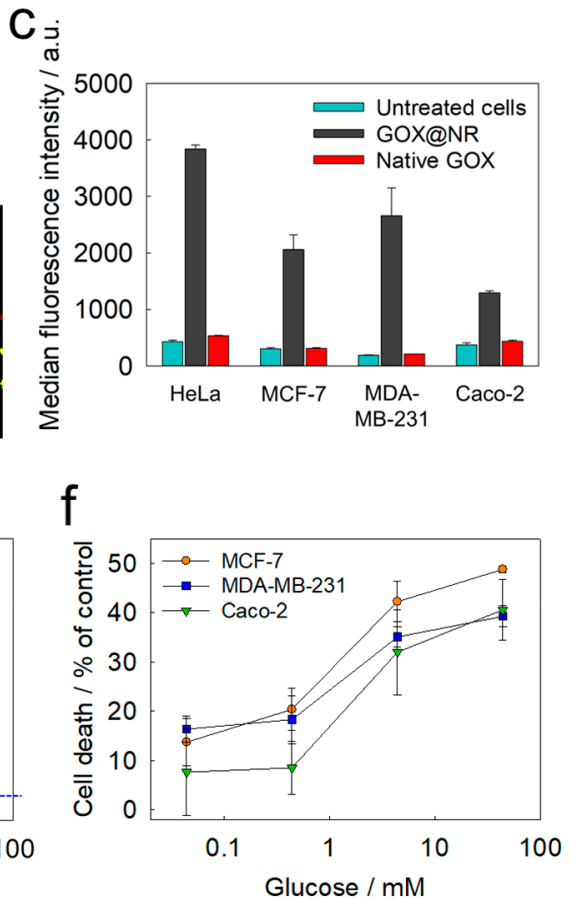

Figure 6. Cytotoxicity of GOX@NR by selective intracellular production of hydrogen peroxide. (a) Concept of the experiment: after glucose depletion (1), GOX@NR is delivered to the cells (2), free GOX@NR are removed (3), and then glucose is added to initiate the GOX reaction (4). (b) Confocal laser microscopic images of the internalized GOX@NR into HeLa cells. Green indicates fluorescein-labeled GOX, and red indicates stained cell membrane. Scale bars represent $10 \mu \mathrm{m}$. (c) Determination of cellular interaction of the GOX@NR and native GOX by flow cytometry $(n=3$, error bars = SD). (d) Cytotoxicity assay of GOX@NR $(n=8$, error bars = SD $)$ and native GOX $(n=5$, error bars = SD $)$-mediated cell death in different concentrations of added glucose for HeLa cells. (e) Hydrogen peroxide production assay of GOX@NR and native GOX ( $n=3$, error bars $=\mathrm{SD})$. Blue dashed line represents intracellular level $(0.2 \mu \mathrm{M})$ of the hydrogen peroxide without treatment of GOX, however, going through $6 \mathrm{~h}$ of glucose starving condition. (f) Cytotoxicity assay of GOX@NR-mediated cell death in different concentrations of glucose for MDAMD-231, MCF-7, and Caco-2 cells $(n=3$, error bars $=\mathrm{SD})$.

undelivered GOX@NR or native GOX was removed before adding glucose into the cells. As shown in Figure 6d, only GOX@NRs proved to have an effective cytotoxicity (HeLa) due to cellular uptake and intracellular production of hydrogen peroxide. In contrast, native GOX did not exhibit a high degree of cytotoxicity, because it was not efficiently attached and delivered to the cells. By intracellular hydrogen peroxide determination assay, we found that the treatment of GOX@ NRs produced additional hydrogen peroxide in relation to the glucose concentration (Figure 6e). The produced hydrogen peroxide increased the intracellular hydrogen peroxide up to a toxic level, leading to selective cell death for the GOX@NRtreated cancer cells. The cellular uptake of GOX@NRs and native GOX was confirmed by confocal laser microscopy, proving a significant amount of GOX@NRs (prepared with fluorescein-labeled GOX, green) on the cellular membrane and inside of the cells (Figure 6b), thereby promoting the intracellular GOX reaction. Due to the polydispersity, the smaller nanoreactors could be transported into the cells by an endocytic pathway, while larger nanoreactors attached to the cellular membrane. Our data proved that the GOX reaction of the nanoreactor is not strong outside the cells, which indicates that they are taken up by the cells. In contrast, no green fluorescence was detected inside the cells when we tried to deliver the native GOX (Figure S8) with low cytotoxicity. We further verified the successful uptake of the GOX@NRs into the cells by flow cytometry, whereas only a negligible amount of native GOX was allowed to be taken up (Figure $6 \mathrm{c}$ and Figure S9). Three model cell lines (MDA-MB-231, human breast cancer; MCF-7, human breast cancer; and $\mathrm{CaCo}-2$, human colorectal cancer) were further investigated. In all cases, similar cellular uptake and cytotoxicity induced by the intracellular delivered nanoreactors were proven (Figure $6 \mathrm{f}$ and Figure S10), indicating that the strategy is applicable to numerous types of cancer cells.

In conclusion, we described oncolytic nanoreactors with loaded glucose oxidase (GOX) to produce hydrogen peroxide intracellularly. We used fluoride-catalyzed sol-gel chemistry to synthesize hollow silica nanoreactors at neutral $\mathrm{pH}$, which preserved the GOX activity, protected GOX against proteolysis, and increased its thermal stability. The GOX nanoreactors were effectively delivered to living cancer cells, in which hydrogen peroxide was produced by the enzyme by consuming glucose in the cytoplasm and eventually led to cell death. Thus, our GOX nanoreactors are a promising platform for enzyme reactions and anticancer therapy inside of cells. To improve the rate of cellular uptake and the tumor accumulation, the development of smaller nanoreactors $(\leq 100 \mathrm{~nm})$ or an active targeting strategy are currently planned in our lab. Glucose levels in deep tumors might be lower than normal physiological conditions due to energetic metabolisms, but our nanoreactors were able to kill the cancer cells at lower glucose levels. By combining other anticancer strategies, synergistic anticancer efficacies might be envisioned. The sol-gel process proceeded under mild conditions and increased the protein stability compared to native proteins. Moreover, enzyme reactions in confinement are known to be more efficient than native enzymes. Therefore, our enzyme- 
loading nanoreactor is thought to be a valuable toolkit for other protein applications ${ }^{34}$ or nanoencapsulation technologies.

\section{ASSOCIATED CONTENT}

\section{S Supporting Information}

The Supporting Information is available free of charge at https://pubs.acs.org/doi/10.1021/acs.nanolett.9b04263.

Materials and experimental details, characterizations of enzymes and nanoreactors, and results of cell experiments for control groups and other cell lines (PDF)

\section{AUTHOR INFORMATION}

\section{Corresponding Authors}

*E-mail: wurm@mpip-mainz.mpg.de.

*E-mail: landfester@mpip-mainz.mpg.de.

\section{ORCID $\odot$}

Frederik R. Wurm: 0000-0002-6955-8489

Katharina Landfester: 0000-0001-9591-4638

\section{Author Contributions}

The manuscript was written through contributions of all authors. S.-M.J., F.R.W., and K.L. conceived the idea. S.-M.J. performed most experiments. F.R.W. and K.L. supervised the project. All authors discussed the results and contributed to cowriting the manuscript. All authors have given approval to the final version of the manuscript.

\section{Notes}

The authors declare no competing financial interest.

\section{ACKNOWLEDGMENTS}

This work is part of the MaxSynBio consortium, which was jointly funded by the Federal Ministry of Education and Research of Germany and the Max Planck Society. We appreciate Dr. Carole Champanhac (MPIP) for assisting flow cytometry and confocal microscopy of the cells. We also thank Dr. Robert Graf (MPIP) for solid-state NMR spectroscopy studies.

\section{REFERENCES}

(1) Chang, F.-P.; Hung, Y.; Chang, J.-H.; Lin, C.-H.; Mou, C.-Y. Enzyme Encapsulated Hollow Silica Nanospheres for Intracellular Biocatalysis. ACS Appl. Mater. Interfaces 2014, 6 (9), 6883-6890.

(2) Lian, X.; Huang, Y.; Zhu, Y.; Fang, Y.; Zhao, R.; Joseph, E.; Li, J.; Pellois, J.-P.; Zhou, H.-C. Enzyme-MOF Nanoreactor Activates Nontoxic Paracetamol for Cancer Therapy. Angew. Chem., Int. Ed. 2018, 57 (20), 5725-5730.

(3) Li, J.; Li, Y.; Wang, Y.; Ke, W.; Chen, W.; Wang, W.; Ge, Z. Polymer Prodrug-Based Nanoreactors Activated by Tumor Acidity for Orchestrated Oxidation/Chemotherapy. Nano Lett. 2017, 17 (11), 6983-6990.

(4) Kim, E.-J.; Bhuniya, S.; Lee, H.; Kim, H. M.; Cheong, C.; Maiti, S.; Hong, K. S.; Kim, J. S. An Activatable Prodrug for the Treatment of Metastatic Tumors. J. Am. Chem. Soc. 2014, 136 (39), 1388813894.

(5) Malekshah, O. M.; Chen, X.; Nomani, A.; Sarkar, S.; Hatefi, A. Enzyme/Prodrug Systems for Cancer Gene Therapy. Current Pharmacology Reports 2016, 2 (6), 299-308.

(6) Niraula, N. P.; Kim, S.-H.; Sohng, J. K.; Kim, E.-S. Biotechnological doxorubicin production: pathway and regulation engineering of strains for enhanced production. Appl. Microbiol. Biotechnol. 2010, 87 (4), 1187-1194.

(7) Guerra-Bubb, J.; Croteau, R.; Williams, R. M. The early stages of taxol biosynthesis: An interim report on the synthesis and identification of early pathway metabolites. Nat. Prod. Rep. 2012, 29 (6), 683-696.

(8) Vilema-Enríquez, G.; quez, G.; Arroyo, A.; Grijalva, M.; AmadorZafra, R. I.; Camacho, J. Molecular and Cellular Effects of Hydrogen Peroxide on Human Lung Cancer Cells: Potential Therapeutic Implications. Oxid. Med. Cell. Longevity 2016, 2016, 12.

(9) Teramoto, S.; Tomita, T.; Matsui, H.; Ohga, E.; Matsuse, T.; Ouchi, Y. Hydrogen Peroxide-Induced Apoptosis and Necrosis in Human Lung Fibroblasts: Protective Roles of Glutathione. Jpn. J. Pharmacol. 1999, 79 (1), 33-40.

(10) Veal, E. A.; Day, A. M.; Morgan, B. A. Hydrogen Peroxide Sensing and Signaling. Mol. Cell 2007, 26 (1), 1-14.

(11) Ahmad, K. A.; Iskandar, K. B.; Hirpara, J. L.; Clement, M.-V.; Pervaiz, S. Hydrogen Peroxide-Mediated Cytosolic Acidification Is a Signal for Mitochondrial Translocation of Bax during Drug-Induced Apoptosis of Tumor Cells. Cancer Res. 2004, 64 (21), 7867.

(12) DiPietrantonio, A. M.; Hsieh, T.-c.; Wu, J. M. Activation of Caspase 3 in HL-60 Cells Exposed to Hydrogen Peroxide. Biochem. Biophys. Res. Commun. 1999, 255 (2), 477-482.

(13) Clément, M.-V.; Ponton, A.; Pervaiz, S. Apoptosis induced by hydrogen peroxide is mediated by decreased superoxide anion concentration and reduction of intracellular milieu. FEBS Lett. 1998, 440 (1-2), 13-18.

(14) Prasetyanto, E. A.; Bertucci, A.; Septiadi, D.; Corradini, R.; Castro-Hartmann, P.; De Cola, L. Breakable Hybrid Organosilica Nanocapsules for Protein Delivery. Angew. Chem., Int. Ed. 2016, 55 (10), 3323-3327.

(15) Thamboo, S.; Najer, A.; Belluati, A.; von Planta, C.; Wu, D.; Craciun, I.; Meier, W.; Palivan, C. G. Mimicking Cellular Signaling Pathways within Synthetic Multicompartment Vesicles with Triggered Enzyme Activity and Induced Ion Channel Recruitment. Adv. Funct. Mater. 2019, 29 (40), 1904267.

(16) Garni, M.; Einfalt, T.; Goers, R.; Palivan, C. G.; Meier, W. Live Follow-Up of Enzymatic Reactions Inside the Cavities of Synthetic Giant Unilamellar Vesicles Equipped with Membrane Proteins Mimicking Cell Architecture. ACS Synth. Biol. 2018, 7 (9), 21162125 .

(17) Rifaie-Graham, O.; Ulrich, S.; Galensowske, N. F. B.; Balog, S.; Chami, M.; Rentsch, D.; Hemmer, J. R.; Read de Alaniz, J.; Boesel, L. F.; Bruns, N. Wavelength-Selective Light-Responsive DASA-Functionalized Polymersome Nanoreactors. J. Am. Chem. Soc. 2018, 140 (25), 8027-8036.

(18) Mason, A. F.; Yewdall, N. A.; Welzen, P. L. W.; Shao, J.; van Stevendaal, M.; van Hest, J. C. M.; Williams, D. S.; Abdelmohsen, L. K. E. A. Mimicking Cellular Compartmentalization in a Hierarchical Protocell through Spontaneous Spatial Organization. ACS Cent. Sci. 2019, 5 (8), 1360-1365.

(19) Li, J.; Dirisala, A.; Ge, Z.; Wang, Y.; Yin, W.; Ke, W.; Toh, K.; Xie, J.; Matsumoto, Y.; Anraku, Y.; Osada, K.; Kataoka, K. Therapeutic Vesicular Nanoreactors with Tumor-Specific Activation and Self-Destruction for Synergistic Tumor Ablation. Angew. Chem., Int. Ed. 2017, 56 (45), 14025-14030.

(20) Zhao, W.; Hu, J.; Gao, W. Glucose Oxidase-Polymer Nanogels for Synergistic Cancer-Starving and Oxidation Therapy. ACS Appl. Mater. Interfaces 2017, 9 (28), 23528-23535.

(21) Zhang, R.; Feng, L.; Dong, Z.; Wang, L.; Liang, C.; Chen, J.; Ma, Q.; Zhang, R.; Chen, Q.; Wang, Y.; Liu, Z. Glucose \& oxygen exhausting liposomes for combined cancer starvation and hypoxiaactivated therapy. Biomaterials 2018, 162, 123-131.

(22) Dinda, S.; Sarkar, S.; Das, P. K. Glucose oxidase mediated targeted cancer-starving therapy by biotinylated self-assembled vesicles. Chem. Commun. 2018, 54 (71), 9929-9932.

(23) Chang, K.; Liu, Z.; Fang, X.; Chen, H.; Men, X.; Yuan, Y.; Sun, K.; Zhang, X.; Yuan, Z.; Wu, C. Enhanced Phototherapy by Nanoparticle-Enzyme via Generation and Photolysis of Hydrogen Peroxide. Nano Lett. 2017, 17 (7), 4323-4329.

(24) Fan, W.; Lu, N.; Huang, P.; Liu, Y.; Yang, Z.; Wang, S.; Yu, G.; Liu, Y.; Hu, J.; He, Q.; Qu, J.; Wang, T.; Chen, X. GlucoseResponsive Sequential Generation of Hydrogen Peroxide and Nitric 
Oxide for Synergistic Cancer Starving-Like/Gas Therapy. Angew. Chem., Int. Ed. 2017, 56 (5), 1229-1233.

(25) Yu, Z.; Zhou, P.; Pan, W.; Li, N.; Tang, B. A biomimetic nanoreactor for synergistic chemiexcited photodynamic therapy and starvation therapy against tumor metastasis. Nat. Commun. 2018, 9 (1), 5044.

(26) Fu, L.-H.; Qi, C.; Hu, Y.-R.; Lin, J.; Huang, P. Glucose OxidaseInstructed Multimodal Synergistic Cancer Therapy. Adv. Mater. 2019, 31 (21), 1808325.

(27) Gaitzsch, J.; Huang, X.; Voit, B. Engineering Functional Polymer Capsules toward Smart Nanoreactors. Chem. Rev. 2016, 116 (3), 1053-1093.

(28) Jo, S.-M.; Wurm, F. R.; Landfester, K. Biomimetic Cascade Network between Interactive Multicompartments Organized by Enzyme-Loaded Silica Nanoreactors. ACS Appl. Mater. Interfaces 2018, 10 (40), 34230-34237.

(29) Barros, L. F.; Kanaseki, T.; Sabirov, R.; Morishima, S.; Castro, J.; Bittner, C. X.; Maeno, E.; Ando-Akatsuka, Y.; Okada, Y. Apoptotic and necrotic blebs in epithelial cells display similar neck diameters but different kinase dependency. Cell Death Differ. 2003, 10 (6), 687697.

(30) Giorgio, M.; Trinei, M.; Migliaccio, E.; Pelicci, P. G. Hydrogen peroxide: a metabolic by-product or a common mediator of ageing signals? Nat. Rev. Mol. Cell Biol. 2007, 8, 722.

(31) Upadhyay, D.; Chang, W.; Wei, K.; Gao, M.; Rosen, G. D. Fibroblast growth factor-10 prevents $\mathrm{H} 2 \mathrm{O} 2$-induced cell cycle arrest by regulation of G1 cyclins and cyclin dependent kinases. FEBS Lett. 2007, 581 (2), 248-252.

(32) Chen, J.-P.; Xu, D. E. G.; Yu, X.-Y.; Zhao, F.-M.; Xu, D.-Q.; Zhang, X.; Cai, B.-C.; Wang, M.-Y. Discrepancy between the effects of morronside on apoptosis in human embryonic lung fibroblast cells and lung cancer A549 cells. Oncol. Lett. 2014, 7 (4), 927-932.

(33) Symons, M. C. R.; Rusakiewicz, S.; Rees, R. C.; Ahmad, S. I. Hydrogen peroxide: a potent cytotoxic agent effective in causing cellular damage and used in the possible treatment for certain tumours. Med. Hypotheses 2001, 57 (1), 56-58.

(34) Choi, J.-M.; Han, S.-S.; Kim, H.-S. Industrial applications of enzyme biocatalysis: Current status and future aspects. Biotechnol. Adv. 2015, 33 (7), 1443-1454. 